\title{
Über das r-Acetacetylpyridyl
}

von

Karl Micko.

Aus dem I. chemischen Laboratorium der k. k. Universität in Wien.

(Vorgelegt in der Sitzung am 2. Juli 1896.)

Im Anschlusse an eine Untersuchung über das $\gamma$-Acetacetylchinolyl ${ }^{1}$ von $\mathrm{H}$. Weidel habe ich versucht, durch Condensation des Pikolinsäureeäthylesters mit Aceton das $\alpha$-Acetacetypyridyl darzustellen. Die Versuche, über deren Resultate ich im Folgenden Bericht erstatte, haben gezeigt, dass diese Verbindung in ausserordentlich glatter Weise durch das nachstehende Verfahren gewonnen werden kann. $30 \mathrm{~g}$ von voll. kommen reinem Pikolinsäureäthylester wurden mit $13.5 \mathrm{~g}$ Aceton (vollständig entwässert) und mit $60 \mathrm{~g}$ Benzol vermischt. Diese Lösung wurde allmälig unter Kühlung auf völlig alkoholfreies Natriumäthylat getropft, welches aus $5.25 \mathrm{~g} \mathrm{Natrium}^{2}$ und absolutem Alkohol hergestellt worden war. Unter ziemlich bedeutender Erwärmung nimmt die Flüssigkeit eine schwach gelbbräunliche Farbe an. Sobald die gesammte Menge des Gemisches eingetragen ist, findet auch eine völlige Auflösung des Natriumäthylats statt. Zur Vollendung der Reaction wird das Ganze noch in ein $40-50^{\circ}$ warmes Wasserbad gestellt und darin etwa eine Stunde belassen. Nach dem Abkühlen beginnt sich die Flüssigkeit zu trüben und es findet Abscheidung eines fein krystallinischen, gelblich gefärbten Niederschlages

1 Siehe die vorstehende Abhandlung.

2 Diese Menge entspricht einem circa $15-20$ procentigen Überschusse. Die Verwendung eines solchen Überschusses hat sich als günstig für die Ausbeute erwiesen. 
statt, der sich so rasch vermehrt, dass die ganze Masse zu einem dicken Brei erstarrt. Derselbe wurde mit circa $250 \mathrm{~cm}^{3}$ Wasser vermischt und so lange mit Kohlensäure behandelt, bis der krystallinische Niederschlag vollständig gelöst war. ${ }^{1}$ Durch wiederholtes Ausschütteln mit Benzol kann nun der Lösung leicht die gesammte Menge des $\alpha$-Acetacetylpyridyls entzogen werden, welches durch Zersetzung der früher abgeschiedenen Natriumverbindung entstanden ist.

Die vereinigten Benzolauszüge hinterlassen nach dem Abdestilliren ein braungefärbtes Ö1, welches beim Abkühlen nach kurzer Zeit krystallinisch erstarrt. Das wieder Verflüssigte habe ich behufs weiterer Reinigung zunächst einige Zeit im Vacuum auf die Temperatur von $80-90^{\circ}$ erwärmt, um die letzten Reste des Benzols zu vertreiben, und dann im Vacuum destillirt. Bei dem Druck von $15 \mathrm{~mm}$ siedet die Flüssigkeit bei $137-143^{\circ} \mathrm{C}$. ohne dass Zersetzung eintritt. Das schwach gelbgefärbte Destillat erstarrt sehr bald; wobei lebhafte Wärmeentwicklung eintritt.

Durch Abpressen des so erhaltenen Krystallkuchens konnte eine kleine Quantität eines öligen Nebenproductes entfernt werden. Nach dem Pressen habe ich die Substanz durch wiederholtes Umkrystallisiren aus Petroläther endlich in völlig reinem Zustande erhalten. Die Verbindung, die sich nach der Gleichung

$$
\mathrm{C}_{5} \mathrm{H}_{4} \mathrm{NCOOC}_{2} \mathrm{H}_{5}+\mathrm{CH}_{3} \cdot \mathrm{CO} . \mathrm{CH}_{3}=\mathrm{C}_{5} \mathrm{H}_{4} \mathrm{NCOCH}_{2} \mathrm{CO} . \mathrm{CH}_{3}+\mathrm{C}_{2} \mathrm{H}_{5} \mathrm{OH}
$$

bildet, entsteht nach dieser Vorschrift in sehr guter Ausbeute und ich habe bei den verschiedenen Darstellungen durchschnittlich $70-74 \%$ der theoretischen Ausbeute erzielt.

Das $\alpha$-Acetacetylpyridyl hat ein ganz aussergewöhnliches Krystallisationsvermögen und kann dasselbe je nach Temperaturen- und Concentrationsverhältnissen in sehr verschiedenen Formen erhalten werden. Eine Lösung der Substanz in Petroläther, welche in der Siedehitze hergestellt war, scheidet die Verbindung in Krystallspiessen ab, die zu Drusen verwachsen sind. Eine derartige, verdünnte Lösung bildet $z$ arte, nadel-

1 Statt Kohlensäure einzuleiten, kann auch die Flüssigkeit mit verdünnter Essigsäure neutralisirt werden. 
förmige Krystalle, die zu flockenförmigen Gebilden angeordnet sind.

Übersättigte Lösungen, welche sich beim ruhigen Stehen durch allmäliges Abdunsten concentrirter Lösungen bei $18^{\circ}$ bis $20^{\circ} \mathrm{C}$. bilden, scheiden das $\alpha$-Acetacetypyridyl in farblosen, durchsichtigen, glasglänzenden Krystallen ab, die wohlausgebildete monokline Pyramiden darstellen oder lange (bis zu $10 \mathrm{~cm}$ ) Prismen bilden.

Herr Dr. P. Heberdey hatte die Liebenswürdigkeit, diese Krystalle einer Untersuchung zu unterziehen und theilt über dieselben Folgendes mit:

$\gg$ Krystallsystem: monoklin.

$$
\begin{aligned}
a: b: c & =0 \cdot 4679: 1: 0 \cdot 4824 . \\
\eta & =82^{\circ} 2 .
\end{aligned}
$$

Formen:

$$
\text { (110), (121), (010), (011). }
$$

Winkelwerthe:

$$
\begin{aligned}
& 110: 110 \ldots \ldots \ldots 49^{\circ} 30^{\prime} \\
& 110: 011 \ldots \ldots \ldots 73 \quad 10 \\
& 011: 011 \ldots \ldots \ldots 4930 \\
& 011: 121 \ldots \ldots \ldots 4344 \\
& 011: 010 \ldots \ldots \ldots 6515 .
\end{aligned}
$$

Der Schmelzpunkt des Acetacetylpyridyls liegt bei $49^{\circ}$ bis $50^{\circ}$ C. (uncorr.). Es wird leicht von Alkohol, Äther, Chloroform, Benzol und siedendem Petroläther in Lösung gebracht. In Wasser ist die Verbindung so gut wie unlöslich. Sie ist, wie schon erwähnt, in höherer Temperatur flüchtig und verdunstet auch in sehr geringer Quantität schon bei gewöhnlicher Temperatur; sie zeigt daher einen schwach aromatischen Geruch.

Die alkoholische Lösung gibt mit Eisenchlorid nach Art der $\beta$-Diketone eine intensiv rothe Reaction, die ähnlich in ihrem Farbenton der Rhodonreaction ist.

Die Analysen der im Exsiccator über Schwefelsäure getrockneten Substanz ergaben Zahlen, aus welchen die Formel $\mathrm{C}_{3} \mathrm{H}_{9} \mathrm{NO}_{2}$ abgeleitet werden konnte. 
I. $0 \cdot 2702 g$ Substanz gaben $0.6553 g$ Kohlensäure und $0.1334 g$ Wasser.

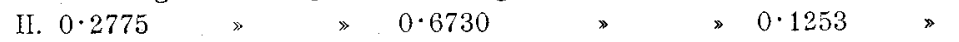

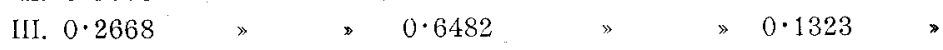

IV. 0.2988 » $23 \mathrm{~cm}^{3}$ feuchten Stickstoff bei $20^{\circ} \mathrm{C}$. und $748 \cdot 9 \mathrm{~mm}$ Druck.

V. $0 \cdot 2986 \mathrm{~g}$ Substanz gaben $23 \mathrm{~cm}^{3}$ feuchten Stickstoff bei $22^{\circ} \mathrm{C}$, und $750 \cdot 2$ mm Druck.

In 100 Theilen:

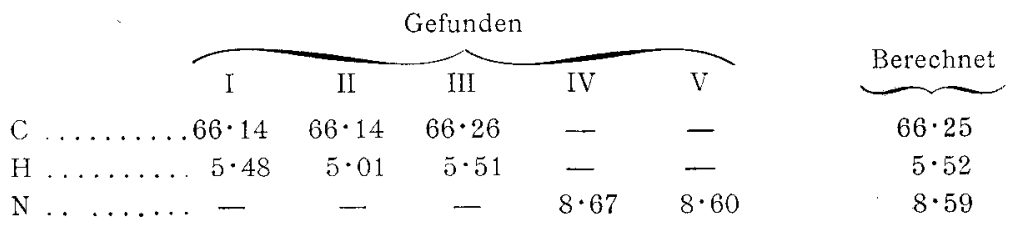

Die angegebene Formel habe ich durch Untersuchung einer Anzahl von Salzen und Doppelverbindungen verificirt.

Salzsaure Verbindung. Dieselbe wird in Form von wasserhellen, hygroskopischen Krystallen erhalten, die sich aus einer Lösung des $\alpha$-Acetacetylpyridyls in verdünnter Salzsäure beim Abdunsten im Vacuum über Ätzkalk und Schwefelsäure ausscheiden. Die Verbindung ist ziemlich zersetzlich. Schon beim Trocknen bei etwa $100^{\circ} \mathrm{C}$. tritt Verfärbung unter Entwicklung stechend riechender Dämpfe ein. Höher erhitzt schwärzt sich die Substanz und schmilzt schliesslich unter Zersetzung. Für die Analyse habe ich die Verbindung zwischen $50^{\circ}$ und $60^{\circ} \mathrm{C}$. im Vacuum getrocknet. Dabei konnte ich die Beobachtung machen, dass sich ein Theil derselben unzersetzt verflüchtigte. Die Chlorbestimmung ergab einen mit der Formel $\mathrm{C}_{9} \mathrm{H}_{9} \mathrm{NO}_{2}+\mathrm{HCl}$ übereinstimmenden Werth.

$0 \cdot 2448 g$ Substanz ergaben $0 \cdot 1780 \mathrm{~g}$ Chlorsilber.

In 100 Theilen:

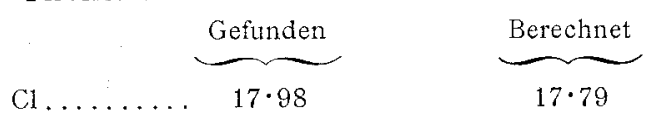

Chloroplatinat. Dasselbe fällt auf Zusatz von Platinchlorid zu einer Lösung der Base in mässig concentrirter Salzsäure sofort in kleinen. Krystallblättchen aus. Die von der Mutterlauge getrennte Ausscheidung habe ich nach dem 
Waschen mit verdünntem Alkohol aus heisser (etwa 15-procentiger) Salzsäure umkrystallisirt. In derselben ist die Verbindung in der Siedehitze ziemlich leicht löslich und krystallisirt in gelbrothen Krystalltafeln, die wasserhältig sind. Da das Chloroplatinat schon bei verhältnissmässig niederer Temperatur unter Dunkelfärbung Zersetzung erfährt, so musste ich dasselbe auch im Vacuum bei $50^{\circ} \mathrm{C}$. trocknen. Die Platin- und Chlorbestimmungen lieferten Zahlen, welche für die Formel $2\left(\mathrm{C}_{9} \mathrm{H}_{9} \mathrm{NO}_{2}+\mathrm{HCl}\right)+\mathrm{PtCl}_{4}$ beweisend sind.

I. $0 \cdot 2080 \mathrm{~g}$ Substanz gaben $0 \cdot 2418 \mathrm{~g}$ Chlorsilber.

II. $0 \cdot 2188 g$ Substanz gaben $0 \cdot 0582 g$ Platin.

In 100 Theilen:

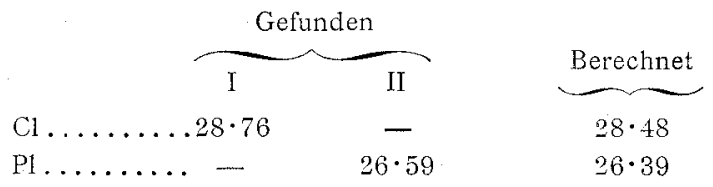

Die lufttrockene Verbindung enthält zwei Moleküle Wasser, wie die folgende Analyse beweist:

I. $0.1818 g$ Substanz gaben $0.0458 g$ Platin.

II. $0 \cdot 1998 \mathrm{~g}$ Substanz gaben $0.2228 \mathrm{~g}$ Chlorsiber.

In 100 Theilen:

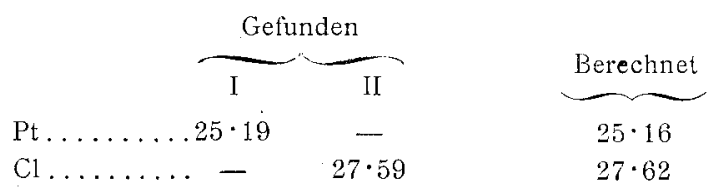

Ich habe durch Vermischen einer Lösung der salzsauren Verbindung mit Goldchlorid versucht, ein Aurichlorat herzustellen. Die Bildung desselben erfolgt, doch findet während des Abdampfens unter Abscheidung von Gold totale Zersetzung statt.

Mit Quecksilberchlorid bildet das Acetacetylpyridyl zwei Doppelverbindungen.

Erste $Q$ uecksilberverbindung. Dieselbe ist nach der Formel $\mathrm{C}_{9} \mathrm{H}_{9} \mathrm{NO}_{2}+\mathrm{HCl}+\mathrm{HgCl}_{2}+2 \mathrm{H}_{2} \mathrm{O}$ zusammengesetzt und 
scheidet sich in kleinen, glänzenden, pyramidenförmigen Krystä1lchen $a b$, wenn eine mit concentrirter Salzsäure stark angesäuerte alkoholische Lösung der Base mit alkoholischer Sublimatlösung versetzt und langsam abdunsten gelassen wird. Die Analyse der lufttrockenen Substanz ergab die mit obiger Formel übereinstimmenden Werthe.

I. $0.2670 \mathrm{~g}$ Substanz gaben $0 \cdot 1050 \mathrm{~g}$ Quecksilber und $0.2311 \mathrm{~g}$ Chlor silber.

II. $0 \cdot 4488 \mathrm{~g}$ Substanz gaben $0 \cdot 1760 \mathrm{~g}$ Quecksilber und $0 \cdot 3868 \mathrm{~g}$ Chlorsilber.

In 100 Theilen:

\begin{tabular}{|c|c|c|c|}
\hline \multicolumn{4}{|c|}{ Gefunden } \\
\hline & $\bar{I}$ & II & Berechnet \\
\hline $\mathrm{Hg}$ & $39 \cdot 32$ & $39 \cdot 21$ & $39 \cdot 48$ \\
\hline $\mathrm{Cl}$. & $21 \cdot 41$ & $21 \cdot 32$ & 21.03 \\
\hline
\end{tabular}

Die lufttrockene Substanz enthält, wie früher angegeben, $z$ wei Moleküle Wasser, welche zwischen $50^{\circ}$ und $60^{\circ} \mathrm{C}$. im Vacuum vollständig entweichen. Die Analyse des bei dieser Temperatur getrockneten Salzes ergab folgendes Resultat:

I. $0 \cdot 2750 \mathrm{~g}$ Substanz gaben $0 \cdot 1154 g$ Quecksilber und $0.2534 \mathrm{~g}$ Chlorsilber.

II. $0 \cdot 4306 g$ Substanz gaben $0 \cdot 1815 g$ Quecksilber und $0 \cdot 3953 g$ Chlorsilber.

In 100 Theilen:

\begin{tabular}{ccc} 
Gefunden & \multicolumn{11}{l}{ Berechnet } \\
$\mathrm{Hg} \ldots \ldots \ldots 41 \cdot 96 \quad 42 \cdot 15$ & $42 \cdot 51$ \\
$\mathrm{C} \ldots \ldots \ldots 22 \cdot 79 \quad 22 \cdot 71$ & $22 \cdot 64$
\end{tabular}

Zweite Quecksilberverbindung. Wird die vorhin beschriebene Quecksilberverbindung aus warmem, verdünntem Alkohol (60-70-procentig) umkrystallisirt, so scheiden sich nicht mehr die pyramidenförmigen Krystalle ab, sondern es bilden sich seidenglänzende, farblose, feine Nadeln. Beim Auflösen muss jedoch ein zu langes Erhitzen vermieden werden, weil sonst eine Abscheidung von Quecksilberchlorür und Quecksilber eintritt. 
Die Quecksilber- und Chlorbestimmung dieses krystallwasserfreien Productes führte zur Formel $\mathrm{C}_{9} \mathrm{H}_{9} \mathrm{NO}_{2}+\mathrm{HgCl}_{2}$.

I. $0 \cdot 3034 g$ Substanz gaben $0 \cdot 1390 g$ Quecksilber und $0 \cdot 2040 \mathrm{~g}$ Chlorsilber.

II. $0 \cdot 2954$

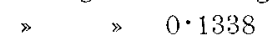

III. $0 \cdot 3903$

$\gg \quad>0.1779$

$\Rightarrow 0 \cdot 1956 \quad$ "
$\Rightarrow 0.2594$ "

In 100 Theilen:

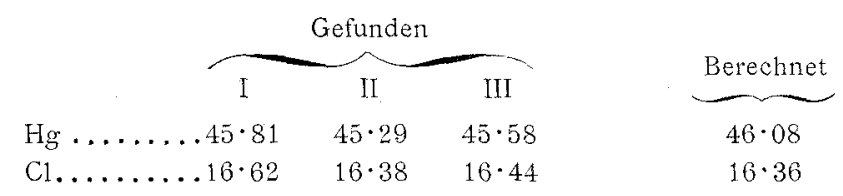

\section{Einwirkung concentrirter Alkalien.}

Beim längeren Erhitzen einer Lösung des $\alpha$-Acetacetylpyridyls in concentrirter Kali- oder Natronlauge auf $100^{\circ} \mathrm{C}$. findet Zersetzung statt. Wird nun die Lösung im Dampfstrom destillirt, so verflüchtigt sich Aceton, welches mit Hilfe der Jodoformreaction durch sein Verhalten gegen ammoniakalische Silberlösung und fuchsinschwefelige Säure als solches erkannt wurde. Im Destillationsrückstand findet sich Pikolinsäure vor, die durch das charakteristische Kupfersalz nachgewiesen wurde. Die Zersetzung findet demnach im Sinne der Gleichung

statt.

$$
\mathrm{C}_{9} \mathrm{H}_{9} \mathrm{NO}_{2}+\mathrm{KOH}=\mathrm{C}_{6} \mathrm{H}_{4} \mathrm{NO}_{2} \mathrm{~K}+\mathrm{C}_{3} \mathrm{H}_{6} \mathrm{O}
$$

\section{Einwirkung von Phenylhydrazin.}

Das $\alpha$-Acetacetylpyridyl charakterisirt sich durch sein Verhalten gegen Phenylhydrazin als ein $\beta$-Diketon, da es wie diese Verbindungen, mit Phenylhydrazin ein als 1-Phenyl-5-Methy3-Pyridylpyrazol zu bezeichnendes Product bildet:

$$
\begin{array}{rl}
\mathrm{C}_{5} \mathrm{H}_{4} \mathrm{COCH}_{2} \mathrm{CO} . \mathrm{CH}_{3}+\mathrm{C}_{6} \mathrm{H}_{5} \mathrm{NH} \cdot \mathrm{NH}_{2}= & \\
=2 \mathrm{H}_{2} \mathrm{O}+\mathrm{C}_{5} \mathrm{H}_{4} \mathrm{C}-\mathrm{CH}=\mathrm{C}-\mathrm{CH}_{3} & \mathrm{~N}-\mathrm{N}-\mathrm{C}_{6} \mathrm{H}_{5} .
\end{array}
$$

Die Darstellung desselben wurde in folgender Weise durchgeführt. Die Lösung von $10 g$ in möglichst wenig verdünnter 
Salzsäure wird mit der berechneten Menge einer Lösung von Phenylhydrazinchlorhydrat versetzt. Nach dem Eintragen von concentrirtem Natriumacetat entsteht sofort die Abscheidung öliger Tröpfchen, welche beim Erwärmen auf dem Wasserbade nach etwa 3 Viertelstunden vollendet ist. Das ausgeschiedene Product besitzt eine dunkelgelbe Farbe und kann seiner Unlöslichkeit in Wasser wegen von der Salzlösung leicht befreit werden. Das Öl löst sich in Äther leicht auf, beim Abdunsten scheidet sich aber nichts Krystallinisches ab. Bei höherer Temperatur ist es, ohne Zersetzung zu erleiden, flüchtig und desshalb konnte ich das Rohproduct durch Destillation (im Vacuum) reinigen. Bei der Temperatur von $215^{\circ} \mathrm{C}$. (bei $15 \mathrm{~mm}$ Druck) destillirt es als gelbliches, dickflüssiges Ö1, das selbst bei $-20^{\circ} \mathrm{C}$. nicht zum Krystallisiren zu bringen war. Dasselbe bräunt sich beim längeren Stehen an der Luft, löst sich weder in Wasser, noch im Petroläther, leicht aber in Alkohol und Äther. Die Analyse ergab Werthe, welche mit den aus der Formel $\mathrm{C}_{15} \mathrm{H}_{13} \mathrm{~N}_{3}$ gerechneten nahezu übereinstimmen.

I. $0 \cdot 2518 \mathrm{~g}$ Substanz gaben $0 \cdot 7012 \mathrm{~g}$ Kohlensäure und $0 \cdot 1180 \mathrm{~g}$ Wasser.

II. $0 \cdot 2060 \mathrm{~g}$ Substanz gaben $32 \cdot 75 \mathrm{~cm}^{3}$ feuchten Stickstoff bei $23^{\circ} \mathrm{C}$. und $744 m m$ Druek.

In 100 Theilen:

\begin{tabular}{|c|c|c|c|}
\hline \multicolumn{3}{|c|}{ Gefunden } & \multirow[b]{2}{*}{ Berechnet } \\
\hline & I & II & \\
\hline$C \ldots \ldots \ldots$ & $75 \cdot 95$ & - & $76 \cdot 59$ \\
\hline$H \ldots \ldots \ldots$ & $5 \cdot 20$ & - & $5 \cdot 53$ \\
\hline$N \ldots \ldots \ldots$ & - & $17 \cdot 52$ & $17 \cdot 87$ \\
\hline
\end{tabular}

Das (1)-Phenyl-(5)-Methyl-(3)-Pyridyl-Pyrazol liefert bei Behandlung mit Jodäthyl offenbar in Folge des Vorhandenseins des Pyridinkernes ein Additionsproduct, welches durch dreistündiges Erhitzen des Pyrazols mit überschüssigem Jodäthyl auf $100-110^{\circ} \mathrm{C}$. im Einschmelzrohr dargestellt werden kann. Nach beendeter Einwirkung ist in den Röhren eine gelbe, blätterig krystallinische Masse abgeschieden, welche in einem dickflüssigen, braunen Öle eingebettet ist. Nach Verflüchtigung des unverbrauchten Jodids wird der Rückstand in siedendem Wasser gelöst. Die Lösung scheidet beim Abdunsten licht- 
citronengelb gefärbte, blätterige Krystallaggreate $a b$, die bei $181-183^{\circ} \mathrm{C}$. unter Zersetzung schmelzen. Die Analyse, sowie die nach der Methode von J. Herzig ${ }^{1}$ ausgeführte Äthylbestimmung ergaben Zahlen, welche mit der Formel

völlig übereinstimmen.

$$
\mathrm{C}_{15} \mathrm{H}_{13} \mathrm{~N}_{3}+\mathrm{JC}_{2} \mathrm{H}_{5}
$$

I. $0.1681 \mathrm{~g}$ Substanz gaben $0.3252 \mathrm{~g}$ Kohlensäure und $0.0660 \mathrm{~g}$ Wasser.

II. $0.1908 \mathrm{~g}$ Substanz gaben $17.75 \mathrm{~cm}^{3}$ feuchten Stickstoff bei $18^{\circ} \mathrm{C}$. und $740 \mathrm{~mm}$ Druck.

III. $01684 \mathrm{~g}$ Substanz gaben $0 \cdot 1001 \mathrm{~g}$ Jodsilber.

IV. $0 \cdot 4836 \mathrm{~g}$ Substanz gaben $0 \cdot 3200 \mathrm{~g}$ Jodsilber.

In 100 Theilen:

\begin{tabular}{|c|c|c|c|c|c|}
\hline & \multicolumn{4}{|c|}{ Gefunden } & \multirow{2}{*}{ Berechnet } \\
\hline & I & II & III & IV & \\
\hline $\mathrm{c} \ldots \ldots \ldots$ & $52 \cdot 76$ & - & - & - & $52 \cdot 17$ \\
\hline $\mathrm{H} \ldots \ldots \ldots$ & $4 \cdot 36$ & - & - & $\ldots$ & $4 \cdot 60$ \\
\hline $\mathrm{N} \ldots \ldots \ldots \ldots$ & - & $10 \cdot 45$ & - & - & $10 \cdot 74$ \\
\hline $\mathrm{s} \ldots \ldots \ldots$ & - & - & $32 \cdot 13$ & - & $32 \cdot 48$ \\
\hline $\mathrm{C}_{2} \mathrm{H}_{5} \ldots \ldots$ & - & - & - & $8 \cdot 16$ & $7 \cdot 41$ \\
\hline
\end{tabular}

Durch Schütteln des Jodids mit frisch gefälltem Chlorsilber entsteht das Chloräthyladditionsproduct, welches sich aus der von Chlorsilber abfiltrirten Lösung beim Einengen im Vacuum in äusserst zerfliesslichen Nadeln ausscheidet. Die Lösung des Chlorhydrats in verdünnter Salzsäure gibt auf Zugabe von Platinchlorid einen feinkörnigen Niederschlag, der nach dem Umkrystallisiren aus Salzsäure in dunkelorangerothen Kryställchen erhalten wurde. Die Verbindung ist, wie die nachfolgende Analyse zeigt, nach der Formel

$$
2\left(\mathrm{C}_{15} \mathrm{H}_{13} \mathrm{~N}_{3}+\mathrm{ClC}_{2} \mathrm{H}_{5}\right) \mathrm{PtCl}_{4}
$$

zusammengesetzt.

I. $0.1112 \mathrm{~g}$ Substanz gaben $0.0231 \mathrm{~g}$ Platin.

II. $0.1304 \mathrm{~g}$ Substanz gaben $0.1209 \mathrm{~g}$ Chlorsilber.

In 100 Theilen:

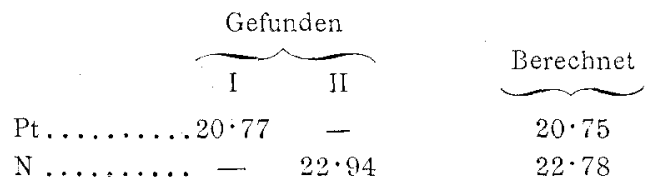

I Monatshefte für Chemie, XV. 


\section{Einwirkung von Hydroxylamin.}

So wie das $\gamma$-Acetacetylchinolyl bei Einwirkung von Hydroxylamin ein von den $\beta$-Diketonen abweichendes Verhalten zeigt, ebenso auch das $\alpha$-Acetacetylpyridyl, welches bei Behandlung mit Hydroxylamin unter verschiedenen Bedingungen bald ein Mono-, bald ein Dioxim zu bilden im Stande ist.

Monoxim. Dasselbe entsteht bei der Einwirkung von Natronhydrat (berechnete Menge) auf die Lösung von $\alpha$-Acetacetylpyridylchlorhydrat und überschüssigem Hydroxylamin.

Da selbst nach 24-stündigem Stehen eine Ausscheidung nicht erfolgte, habe ich die Lösung mit Äther ausgeschüttelt, welcher das Oxim ziemlich leicht und vollständig aufnimmt. Der Äther hinterliess nach dem Abdunsten einen schwach gelblich gefärbten Syrup, der erst nach einigen Wochen beim Verweilen im Exsiccator zu einem Krystallmagma erstarrte. Das Oxim ist in Benzol löslich und scheidet sich aus dieser Lösung auf Zusatz von Petroläther nach einiger Zeit in farblosen Krystallen ab, die leicht in Alkohol, Äther, Chloroform und heissem Wasser löslich sind. Der Schmelzpunkt der wiederholt umkrystallisirten Verbindung wurde $\mathrm{zu} 78^{\circ} \mathrm{C}$. (uncorr.) gefunden. Auf höhere Temperatur erhitzt $\left(150^{\circ} \mathrm{C}\right.$.) verändert sich die Substanz nicht. Die Analysen ergaben Zahlen, welche mit den aus der Formel $\mathrm{C}_{9} \mathrm{H}_{10} \mathrm{~N}_{2} \mathrm{O}_{2}$ gerechneten in guter Übereinstimmung stehen.

I. $0 \cdot 1474 \mathrm{~g}$ Substanz gaben $0.3290 \mathrm{~g}$ Kohlensäure und $0.0764 \mathrm{~g}$ Wasser.

II. $0.1470 \mathrm{~g}$ Substanz gaben $20 \mathrm{~cm}^{2}$ feuchten Stickstoff bei $16^{\circ} \mathrm{C}$. und $756 \mathrm{~mm}$ Druck.

In 100 Theilen:

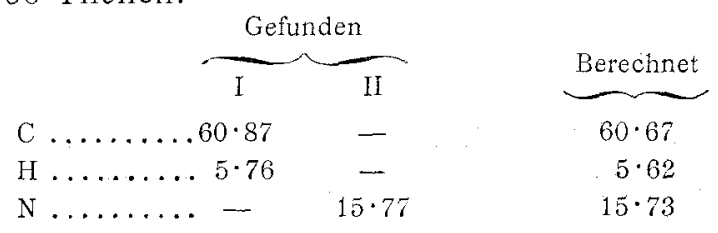

Wird das Monoxim in der von Beckmann beschriebenen Weise $^{1}$ mit Essigsäure, die mit Chlorwasserstoff gesättigt

I Siehe Berl. Ber., 1894, Bd. I. 
wurde, zusammengebracht, so findet bei längerer Einwirkung die Bildung eines neuen Productes statt, welches eine, wie die Untersuchung gezeigt hat, von den bisher erhaltenen Umlagerungsproducten verschiedene Zusammensetzung zeigt. Die Reaction vollendet sich nach einigen Tagen. Destillirt man dann den Uberschuss der Essigsäure im Vacuum ab, so hinterbleibt ein Rückstand, der nach dem Abkühlen krystallinisch erstarrt. Derselbe wird im Wasser gelöst und mit Natriumbicarbonat versetzt und hierauf mit Äther ausgeschüttelt. Nach dem Verjagen desselben hinterbleibt ein in der Kälte erstarrendes Ö1. Durch Umkrystallisiren der Ausscheidung aus siedendem Petroläther (eventuell unter Anwendung von Thierkohle) werden kleine, zu Drusen verwachsene Prismen erhalten, deren Schmelzpunkt bei $48^{\circ}$ C. (uncorr.) liegt. Die Verbindung, welche mit Chlorwasserstoff ein sehr zerfliessliches Salz liefert, ist in Wasser unlöslich, schwerlöslich in Petroläther, dagegen leicht in Alkohol, Äther, Benzol. Die Verbindung ist, wie die Analyse zeigt, nach der Gleichung

$$
\mathrm{C}_{9} \mathrm{H}_{9} \mathrm{NO} . \mathrm{NOH}-\mathrm{H}_{2} \mathrm{O}=\mathrm{C}_{9} \mathrm{H}_{8} \mathrm{~N}_{2} \mathrm{O}
$$

entstanden und es kann die Bildung dieses Körpers durch Wasserabspaltung erfolgen, ohne dass eine Umlagerung in dem Beckmann'schen Sinne eingetreten ist. Die Zusammensetzung der Verbindung kann wohl durch das Schema

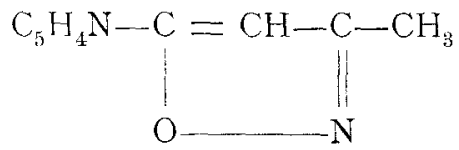

ausgedrückt werden und wäre demnach als ein Isoxazolderivat zu betrachten.

Aus Mangel an Material konnte ich nicht feststellen, welche der beiden CO-Gruppen im Monoxim substituirt wurde.

Die Analyse der im Vacuum getrockneten Substanz ergab:

I. $0.2426 \mathrm{~g}$ Substanz gaben $0.6020 \mathrm{~g}$ Kohlensäure und $0.1058 \mathrm{~g}$ Wasser.

II. $0 \cdot 1684 \mathrm{~g}$ Substanz gaben $26 \cdot 25 \mathrm{~cm}^{3}$ feuchten Stickstoff bei $18^{\circ} \mathrm{C}$. und $745 \cdot 5 \mathrm{~mm}$ Druck. 
In 100 Theilen:

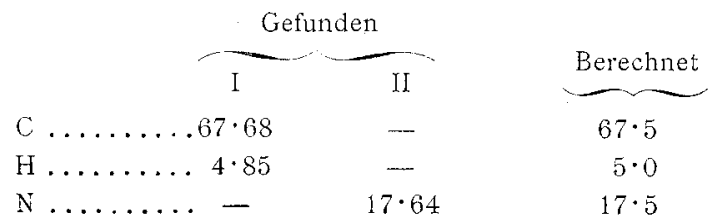

Das Oxim hat die Fähigkeit, mit Phenylhydrazin zu reagiren und gibt ein dickflüssiges Hydrazon, auf dessen nähere Untersuchung ich vorläufig nicht eingegangen bin.

Dioxim. Das Dioxim entsteht durch Behandlung des Monoxims mit einem Überschuss von Hydroxylamin bei Gegenwart von freier Salzsäure. Behufs Darstellung habe ich die Lösung des Monoxims, ohne erst die Natriumsalze zu entfernen, mit Salzsäure schwach angesäuert und hierauf mit einem Überschuss von salzsaurem Hydroxylamin versetzt. Nach circa 8 Tagen war die Lösung schwach gefärbt und es begann endlich bei weiterem Stehen die Abscheidung kleiner, schwach röthlichgefärbter, glänzender Kryställchen. Als nach 14 Tagen eine Vermehrung dieser Abscheidung nicht mehr zu beobachten war, wurde abfiltrirt und die Ausscheidung aus heissem Wasser umkrystallisirt. Beim Auskühlen dieser Lösung bildet sich ein grobkrystallinisches, zart rosenroth gefärbtes Product, welches leicht durch Alkohol, schwer durch siedenden Äther in Lösung gebracht werden kann. Benzol und Petroläther nehmen die Verbindung nicht auf. Unter dem Mikroskope betrachtet, erscheinen die Krystalle als schmale, vierseitige Prismen.

Der Schmelzpunkt liegt zwischen $146^{\circ}$ und $147^{\circ} \mathrm{C}$. (uncorr.). Wenige Grade über diese Temperatur erhitzt (auf $150^{\circ} \mathrm{C}$.) tritt unter Gasentwicklung Zersetzung der Substanz ein. Die Analysen geben Werthe, aus welchen sich die Formel

ableiten lässt.

$$
\mathrm{C}_{5} \mathrm{H}_{4} \mathrm{NCNOHCH}_{2} \cdot \mathrm{CNOH} \cdot \mathrm{CH}_{3}
$$

I. $0 \cdot 2246 g$ Substanz gaben $0 \cdot 4618 g$ Kohlensäure und $0.1149 g$ Wasser.

II. $0.2004 \mathrm{~g}$ Substanz gaben $38.5 \mathrm{~cm}^{3}$ feuchten Stickstoff bei $17^{\circ} \mathrm{C}$. und $741 \mathrm{~mm}$ Druck.

III. $0.1512 \mathrm{~g}$ Substanz gaben $29.3 \mathrm{~cm}^{3}$ feuchten Stickstoff bei $18^{\circ} \mathrm{C}$, und $741 \mathrm{~mm}$ Druck. 
In 100 Theilen:

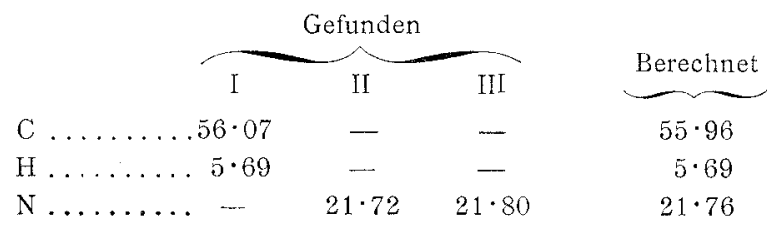

Bei dem Versuch, das Dioxim mit chlorwasserstoffhaltiger Essigsäure umzulagern, habe ich ein auffallendes Resultat erzielt, welches ich in Kürze mittheilen will. Bei längerem Stehen der Lösung des Dioxims mit HCl-haltiger Essigsäure bildet sich ein Körper, dessen Darstellung ich in derselben Weise, wie es bei dem Umwandlungsproducte des Monoxims beschrieben wurde, vorgenommen. Der Rückstand, welcher nach dem Abdestilliren der Ätherauszüge erhalten wurde, liefert beim Umkrystallisiren aus kaltem Petroläther, in welchem sich die Substanz ungemein leicht löst, kleine, glänzende, prismenartige Krystalle, deren Schmelzpunkt bei $37^{\circ} 5 \mathrm{C}$. liegt. Die Analyse dieses Körpers liefert Zahlen, welche mit den aus der Formel $\mathrm{C}_{9} \mathrm{H}_{3} \mathrm{~N}_{2} \mathrm{O}$ berechneten übereinstimmen.

I. $0.2328 \mathrm{~g}$ Substanz gaben $0.5756 \mathrm{~g}$ Kohlensäure und $0.0988 \mathrm{~g}$ Wasser.

II. $0.1508 \mathrm{~g}$ Substanz gaben $23.5 \mathrm{~cm}^{3}$ feuchten Stickstoff bei $19^{\circ} \mathrm{C}$. und $755 \mathrm{~mm}$ Druck.

In 100 Theilen:

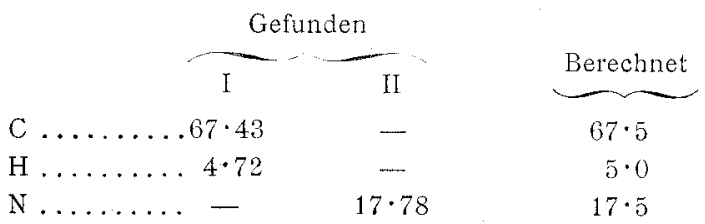

Das zugehörige salzsaure Salz krystallisirt leicht aus der mässig concentrirten wässerigen Lösung in Form von büschelförmig gruppirten Nadeln.

Demnach liefern die beiden Oxime (Mono- und Dioxim) bei Behandlung mit $\mathrm{H} \mathrm{Cl}$-haltiger Essigsäure isomere Producte, welche in ihrem Verhalten wesentliche Verschiedenheit zeigen.

Nicht nur, dass eine Schmelzpunktdifferenz von $11^{\circ} 5 \mathrm{C}$. zwischen diesen beiden Verbindungen besteht, so sind auch 
die Löslichkeiten sehr different. Das Umlagerungsproduct des Monoxims ist in Petroläther schwer löslich, während das des Dioxims in Petroläther äusserst leicht löslich ist. Ebenso sind die zugehörigen salzsauren Salze sowohl in ihrem Aussehen, als auch in ihrer Löslichkeit in Wasser verschieden. Beide zeigen sich aber gegen Salzsäure sehr beständig. Der Unterschied dieser isoxazolartig constituirten, isomeren Umwandlungsproducte könnte durch die folgenden Schemata

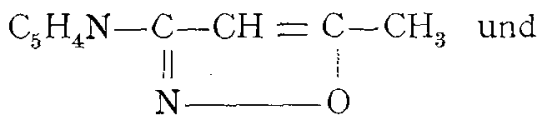<smiles>Cc1cc(Nc2ccccc2)on1</smiles>

zum Ausdruck gebracht werden.

\section{Einwirkung von Ammoniak.}

Gleich dem $\gamma$-Acetacetylchinolyl vermag das $\alpha$-Acetacetylpyridyl leicht Ammoniak zu addiren und ein Aminoacetacetylpyridyl zu liefern. Leitet man in die alkoholische Lösung des Diketons Ammoniak ein, so fällt ein fein krystallinischer Niederschlag aus, der sich als eine Ammoniumverbindung

$$
\mathrm{C}_{5} \mathrm{H}_{4} \mathrm{~N}-\mathrm{CO} . \mathrm{CH}\left(\mathrm{NH}_{4}\right)-\mathrm{CO}-\mathrm{CH}_{3}
$$

erweist. Dieselbe ist aber äusserst unbeständig. Beim Trennen dieses Niederschlages von der Flüssigkeit verflüchtigt sich das Ammoniak und es hinterbleibt unverändertes $\alpha$-Acetacetylpyridyl. Als ich eine Lösung des Diketons in alkoholischem Ammoniak (bei $-20^{\circ} \mathrm{C}$. gesättigt) im Einschmelzrohr während drei Stunden auf $110-120^{\circ}$ erhitzt hatte, zeigte sich in der Röhre keine krystallinische Ausscheidung; erst nach circa 24 Stunden begann die Abscheidung von kleinen, achtseitigen Pyramiden, welche nach dem Umkrystallisiren aus Alkohol monokline, tafelförmige Krystalle lieferten, die nicht selten eine beträchtliche Grösse erreichten. Herr Dr. Heberdey war so freundlich, auch diese Verbindung einer Untersuchung zu unterziehen und theilt mir hierüber Folgendes mit:

$\gg$ Krystallsystem: monosymmetrisch.

$$
\begin{aligned}
a: b: c & =0 \cdot 7004: 1: 0 \cdot 9785 . \\
\eta & =71^{\circ} 54 .
\end{aligned}
$$


Formen: (100), (010), (110), (210), (011), (111), (001).

Die wichtigsten Winkelwerthe sind:

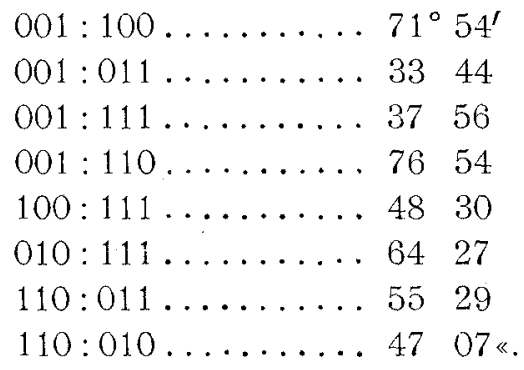

Die Aminoverbindung ist in Alkohol und siedendem Wasser leicht, in Äther schwer, in Benzol und Ligroin aber völlig unlöslich. Sie schmilzt bei der Temperatur von $149^{\circ}$ bis $150^{\circ} \mathrm{C}$. unter theilweiser Zersetzung. Die Verbindung reagirt sowohl mit Phenylhydrazin, als auch mit Hydroxylamin, was durch das Vorhandensein einer CO-Gruppe erklärlich ist.

Mit Rücksicht auf dieses Verhalten und in Hinblick auf die Analyse lässt sich annehmen, dass die Verbindung nach der Forme1

constituirt ist.

$$
\mathrm{C}_{5} \mathrm{H}_{4} \mathrm{NCOCH}=\underset{\mathrm{NH}_{2}}{\mathrm{C}-\mathrm{CH}_{3}}
$$

I. $0 \cdot 2308 g$ Substanz gaben $0.5624 g$ Kohlensäure und $0.1264 g$ Wasser.

II. $0 \cdot 1430 \mathrm{~g}$ Substanz gaben $21.2 \mathrm{~cm}^{3}$ feuchten Stickstoff bei $13^{\circ} \mathrm{C}$. und $750 \mathrm{~mm}$ Druck.

In 100 Theilen:

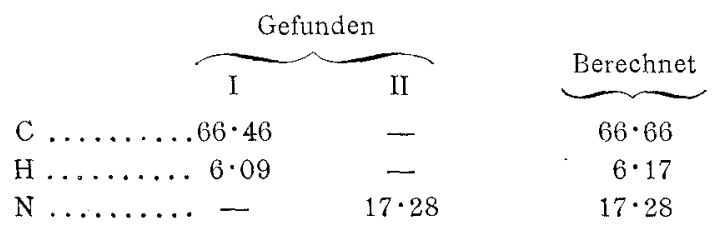

Die beschriebene Aminoverbindung ist ziemlich zersetzlich und liefert keine salzartigen Verbindungen. Wird die Lösung derselben in verdünnter Salzsäure bei gewöhnlicher Temperatur abdunsten gelassen, so scheiden sich aus der 
Flüssigkeit Salmiakkrystalle aus, während in der Lösung die chlorwasserstoffsaure Verbindung des $\alpha$-Acetacetylpyridyls hinterbleibt.

\section{Einwirkung von Wasserstoff im Entstehungszustande.}

Durch nascirenden Wasserstoff kann das $\alpha$-Acetacetylpyridyl verschiedene Producte liefern, und $z$ war war die Bildung eines im Pyridinring hydrirten zweiatomigen Alkohols zu erwarten gewesen. Ferner liegt die Möglichkeit vor, dass beide oder nur eine der $\mathrm{CO}-\mathrm{Gruppen}$ in die $(\mathrm{CHOH})$-Gruppe übergeführt wird. Die verschiedenen Reductionsmittel, die ich in Anwendung gebracht habe, verhalten sich sehr different. Bei Anwendung von Natrium auf die alkoholische Lösung des Diketons in der von Ladenburg beschriebenen Weise ${ }^{1}$ entstehen leicht verharzende, äusserst luftempfindliche Producte, die allen Reinigungsversuchen widerstehen. Ebensowenig führt die Einwirkung von Natriumamalgam oder die Einwirkung von Zinn und Salzsäure zu brauchbaren Producten. Ähnliches gilt auch von Aluminiumamalgam. Zink und verdünnte Essigsäure hingegen verwandeln das $\alpha$-Acetacetylpyridyl im Sinne der Gleichung

$$
\begin{gathered}
\mathrm{C}_{5} \mathrm{H}_{4} \mathrm{~N}-\mathrm{CO}-\mathrm{CH}_{2}-\mathrm{CO}-\mathrm{CH}_{3}+\mathrm{H}_{2}= \\
=\mathrm{C}_{5} \mathrm{H}_{4} \mathrm{~N}-\underset{\mathrm{OH}}{\mathrm{CH}-\mathrm{CH}_{2}-\mathrm{CO}-\mathrm{CH}_{3}} \\
\stackrel{\mathrm{OH}}{1}
\end{gathered}
$$

in einen Ketoalkohol. Am zweckmässigsten mit Rücksicht auf die Ausbeute erweist sich das folgende Verfahren: Je $5 \mathrm{~g}$ des Diketons wurden in $50 \mathrm{~g}$ Eisessig gelöst, mit $250 \mathrm{~cm}^{3}$ Wasser verdünnt; hierauf werden allmälig $10 \mathrm{~g}$ Zinkstaub in die Lösung eingetragen. Anfangs nimmt man die Einwirkung bei gewöhnlicher Temperatur vor; späterhin wird durch mässiges Erwärmen am Wasserbade die Reaction zu Ende geführt. Sowie alles Zink aufgelöst ist, wird die Flüssigkeit etwa auf $1 \frac{1}{2} l$ verdünnt und mit Schwefelwasserstoff gefällt. Das vom Schwefelzink ablaufende, fast farblose Filtrat wird nun im Vacuum abdestillirt.

1 Ann. der Chemie, 247. 
Der braungefärbte Destillationsrückstand beginnt nach dem Abkühlen zu krystallisiren. Diese krystallinische Masse wurde direct mit siedendem Petroläther (dem etwa 20\% Benzol zugesetzt worden war) extrahirt. Nach dem Abdestilliren des Lösungsmittels bildeten sich farblose Nadeln, die nach dem Reinigen einen Schmelzpunkt von $74^{\circ} \mathrm{C}$. zeigten. Wasser, Alkohol, Äther, Essigsäureester nehmen diese Verbindung leicht auf; schwer ist dieselbe in Petroläther löslich. Aus den Analysen der im Vacuum getrockneten Substanz konnte die Formel $\mathrm{C}_{9} \mathrm{H}_{11} \mathrm{NO}_{2}$ abgeleitet werden.

I. $0.2480 \mathrm{~g}$ Substanz gaben $0.5960 \mathrm{~g}$ Kohlensäure und $0.1460 \mathrm{~g}$ Wasser. II. $0 \cdot 2526 g$ Substanz gaben $0.6072 g$ Kohlensäure und $0 \cdot 1495 g$ Wasser.

In 100 Theilen:

\begin{tabular}{|c|c|c|}
\hline \multicolumn{2}{|c|}{ Gefunden } & \multirow[b]{2}{*}{ Berechnet } \\
\hline I & II & \\
\hline$\ldots \ldots \ldots 65 \cdot 54$ & $65 \cdot 56$ & $65 \cdot 45$ \\
\hline $\mathrm{H} \ldots \ldots \ldots 6.54$ & 6.57 & $6 \cdot 67$ \\
\hline
\end{tabular}

Zur Bestätigung der gegebenen Formel habe ich das Chloroplatinat dargestellt, da die salzsaure Verbindung ihrer Zerfliesslichkeit halber sich iür die Analyse weniger gut eignet.

Chloroplatinat. Dasselbe bildet schön orangerothe Krystalle, die in Wasser ziemlich leicht löslich sind. Die Verbindung scheidet sich daher nur aus einer sehr concentrirten, mit Platinchlorid versetzten Lösung des Chlorhydrats des Ketoalkohols bei längerem Stehen im Exsiccator ab. Das durch Umkrystallisiren aus verdünnter Salzsäure erhaltene wasserfreie Platindoppelsalz ergab bei der Analyse Zahlen, welche für die Formel $2\left(\mathrm{C}_{9} \mathrm{H}_{11} \mathrm{NO}_{2}+\mathrm{HCl}\right)+\mathrm{PtCl}_{4}$ beweisend sind.

I. $0 \cdot 1928 g$ Substanz gaben $0.0506 g$ Platin.

II. $0 \cdot 2314 g$ Substanz gaben $0 \cdot 2690 \mathrm{~g}$ Chlorsilber.

In 100 Theilen:

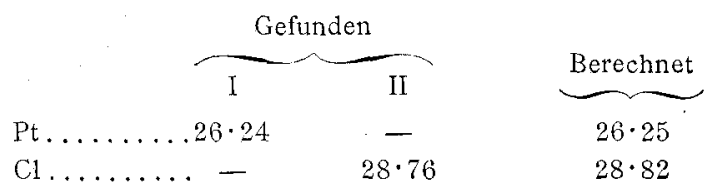


Für die Constitution dieses Reductionsproductes erscheinen folgende Thatsachen beweisend:

1. Das Verhalten desselben gegen Hydroxylamin;

2. der Umstand, dass bei der Oxydation Pikolinsäure neben Essigsäure gebildet wird, und

3. dass durch Alkalien eine Rückbildung von Pikolinsäure nicht erfolgt.

1. Einwirkung von Hydroxylamin. Vermischt man die wässerige Lösung des Ketoalkohols mit Hydroxylamin und fügt hierauf so lange Natriumcarbonatlösung hinzu, als noch Kohlensäureentwicklung $\mathrm{zu}$ beobachten ist, so scheidet sich nach circa 24 Stunden eine reichliche Menge eines kleinkrystallinischen Niederschlages ab. Sowie eine Vermehrung dieser Ausscheidung nicht mehr eintrat, wurde das Ganze mit Äther, der die neue Verbindung ausserordentlich leicht !öst, ausgeschüttelt. Die ätherische Lösung hinterliess nach dem Abdunsten einen schwach gelblichgefärbten Syrup, der nach kurzer Zeit krystallinisch erstarrte. Diese Ausscheidung in Benzol aufgenommen, bildet beim allmäligen Concentriren der Lösung kleine Prismen, welche in Alkohol und Äther leicht, schwieriger in Wasser, kaum aber in Petroläther löslich sind. Die reine Verbindung schmilzt bei $120^{\circ} \mathrm{C}$. und ergab einen Stickstoffgehalt, der mit jenem, welcher sich aus der Formel $\mathrm{C}_{9} \mathrm{H}_{12} \mathrm{~N}_{2} \mathrm{O}_{2}$ berechnet, vollständig übereinstimmt.

$0.1949 \mathrm{~g}$ Substanz gaben $27 \mathrm{~cm}^{3}$ feuchten Stickstoff bei $19^{\circ} \mathrm{C}$. und $758.6 \mathrm{~mm}$ Druck.

In 100 Theilen:

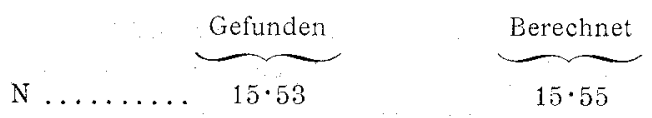

Durch die Bildung dieses Oxims ist das Vorhandensein einer CO-Gruppe in diesem Ketoalkohol unwiderleglich festgestellt. Dieses Resultat findet auch Bestätigung durch die Bildung eines Phenylhydrazons, welches bei Einwirkung von Phenylhydrazin entsteht.

2. Einwirkung von Kaliumpermanganat. Wird in die auf $70^{\circ} \mathrm{C}$. erwärmte wässerige Lösung des Ketoalkohols, 
Kaliumpermanganat in der durch die Gleichung $3 \mathrm{C}_{9} \mathrm{H}_{9} \mathrm{NO}_{2}+8 \mathrm{KMnO}_{4}=\underbrace{3 \mathrm{C}_{6} \mathrm{H}_{4} \mathrm{KNO}_{2}}_{\text {pikolinsaures Kali }}+3 \mathrm{C}_{2} \mathrm{H}_{3} \mathrm{KO}_{2}+\mathrm{K}_{2} \mathrm{CO}_{3}+$ $+2 \mathrm{CO}_{2}+8 \mathrm{MnO}_{2}+3 \mathrm{H}_{2} \mathrm{O}$

ausgedrückten Mengen allmälig eingetragen, so findet anfänglich sofort Entfärbung und Ausscheidung von Mangansuperoxyd statt. Gegen das Ende der Reaction muss ziemlich lange erhitzt werden, bis das Permanganat entfärbt wird. Die vom Braunstein abfiltrirte Lösung wurde genau mit Schwefelsäure neutralisirt und auf dem Wasserbade zur Trockene eingedampft. Der Salzrückstand gab beim Extrahiren mit Alkohol aus denselben pikolinsaures Kali ab, aus welchem durch Kupferacetat das prächtig lazurblau krystallisirende, pikolinsaure Kupfer abgschieden wurde.

In dem umkrystallisirten Kupfersalz habe ich zum Überfluss noch eine Kupferbestimmung vorgenommen. Dieselbe ergab folgendes, die Identität beweisendes Resultat:

$0 \cdot 2198 g$ Substanz gaben $0 \cdot 0561 g$ Kupferoxyd.

In 100 Theilen:

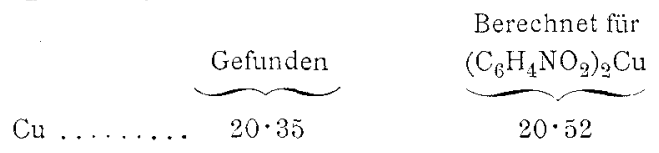

3. Verhalten gegen Alkalien. Wie ich eingangs mitgetheilt habe, zerlegt sich das $\boldsymbol{\alpha}$-Acetacetylpyridyl bei längerer Einwirkung von concentrirten Alkalien in Pikolinsäure und Aceton. Behandelt man in gleicher Weise den in Rede stehenden Ketoalkohol, so findet weder Abspaltung von Aceton, noch Bildung von Pikolinsäure statt. Die Verbindung wird vielmehr bei dieser Behandlung in eine harzige, braungelbe Masse verwandelt, die weder in Wasser, noch in Alkalien löslich ist und aus welcher charakterisirbare Producte nicht abgeschieden werden konnten. Die so leicht nachweisbare Pikolinsäure hat sich bestimmt nicht gebildet. Durch dieses Verhalten scheint mir sichergestellt, dass die in $\alpha$-Stellung befindliche mit dem Pyridinring direct verbundene CO-Gruppe bei der Bildung des 
Ketoalkohols in die $\mathrm{CHOH}-G r u p p e$ verwandelt wurde, denn im gegentheiligen Falle müsste bei der Einwirkung von Alkalien unbedingt Pikolinsäure gebildet werden.

Zum Schlusse komme ich einer angenehmen Pflicht nach, indem ich an dieser Stelle meinem hochverehrten Lehrer, Herrn Prof. H. Weidel für seine, mir während meiner Arbeiten am obgenannten Institute stets $\mathrm{zu}$ Theil gewordene allseitige Unterstützung meinen verbindlichsten Dank ausdrücke.

Ebenso danke ich Herrn Dr. P. He berd ey für die liebenswürdige Übernahme der krystallographischen Untersuchungen. 